\title{
Hybridization of Institutions
}

\author{
Manuel A. Martins ${ }^{1}$, Alexandre Madeira ${ }^{2,1,4}$, \\ Răzvan Diaconescu ${ }^{3}$, Luís S. Barbosa ${ }^{2}$ \\ 1 Department of Mathematics, University of Aveiro ${ }^{1}$ \\ 2 Department of Informatics, Minho University \\ 3 Institute of Mathematics "Simion Stoilow" of the Romanian Academy \\ ${ }^{4}$ Critical Software S.A., Portugal
}

\begin{abstract}
Modal logics are successfully used as specification logics for reactive systems. However, they are not expressive enough to refer to individual states and reason about the local behaviour of such systems. This limitation is overcome in hybrid logics which introduce special symbols for naming states in models. Actually, hybrid logics have recently regained interest, resulting in a number of new results and techniques as well as applications to software specification.

In this context, the first contribution of this paper is an attempt to 'universalize' the hybridization idea. Following the lines of [16], where a method to modalize arbitrary institutions is presented, the paper introduces a method to hybridize logics at the same institution-independent level. The method extends arbitrary institutions with Kripke semantics (for multi-modalities with arbitrary arities) and hybrid features. This paves the ground for a general result: any encoding (expressed as comorphism) from an arbitrary institution to first order logic $(\mathcal{F O} \mathcal{L})$ determines a comorphism from its hybridization to $\mathcal{F O} \mathcal{L}$. This second contribution opens the possibility of effective tool support to specification languages based upon logics with hybrid features.
\end{abstract}

\section{Introduction}

Modern societies are increasingly dependent on software systems and services whose reliability is crucial for their own development, security, privacy, and quality of life. On the other hand, software is large and complex, deals with a multitude of different concerns and has to meet requirements formulated (and verified) at different abstraction levels. For the last three decades such has put forward a research agenda on mathematically sound development methods that seems to be finally emerging as a key concern for industry.

Typically, three issues in this agenda need to be rigorously addressed. The first concerns the sort of mathematical structures suitable to model software systems; the second focus on the languages in which such models can be specified and, finally, the last one addresses the satisfaction relation between the (semantic) mathematical structures and the (syntactic) formulation of requirements as sentences in the specification language. A fourth concern, which is 
becoming more and more relevant in practice, should be added: the fact that the working software engineer has often to capture and relate different kinds of requirements entails the need for a uniform specification framework in which different formalisms can be expressed and related. A quite canonical way to answer this challenge resorts to the notion of institution $[17,14]$ which, as an abstract representation of a logical system, encompasses syntax, semantics and satisfaction, and provides a formal framework for relating, comparing and combining specification logics.

Institution theory [17] is a categorical abstract model theory that arose about three decades ago within specification theory as a response to the explosion in the population of logics in use there, its original aim being to develop as much computing science as possible in a general uniform way independently of particular logical systems. This has now been achieved to an extent even greater than orginally thought, as institution theory became the most fundamental mathematical theory underlying algebraic specification theory, also being increasingly used in other area of computer science. Moreover, institution theory constitutes a major trend in the so-called 'universal logic' (in the sense envisaged by JeanYves Béziau) which is considered by many a true renaissance of mathematical logic.

Modal logics have been successfully used as specification languages for state transition systems, which, on their turn, are taken as basic, underlying structures in program development. From a proof theoretic point of view, such logics have interesting algorithmic proprieties, and, moreover, they can naturally be translated to first order logic. However, (non-hybrid) modal logics do not allow explicit references to specific states of the underlying transition system which, in a number of cases, is a desirable feature in a specification. For instance, such modal logics are adequate to specify systems as dynamic processes which evolve in response to events. But, on the other hand, they are not expressive enough to identify particular states in a system's evolution, neither to express (local) properties referring to one such state or a group thereof. Hybrid logic [2], on the other hand, overcomes this limitation by introducing nominals as references to specific states in a modal framework, taking together features from first-order logic and modal logic.

Historically, hybrid logic was introduced by Arthur Prior [23] in the 50's. Afterwards, his student Robert Bull spread out the theory significantly by establishing a number of completeness results for generalizations of Prior's hybrid logic. After a period without much developments, in the 80's the Bulgarian school of logic (namely Passy, Tinchev, Gargov and Goranko) recovered the interest in hybrid logic, studying, in particular, the possible roles of the binder operator [22]. More recently, Areces and Blackburn intensely expanded up the theory (cf. the dedicated web page at http://hylo.loria.fr/), addressing, notably, the complexity of the satisfiability problem. The work of Braüner on proof theory for hybrid logic should also be mentioned [5]. His study of quantified hybrid logic is, in a sense, at the origin of the results presented in this paper. Actually, the way first order and hybrid logics are combined in quantified hybrid logic, was a first 
motivation for the quest for a general, institution-independent approach to the hybridization of logics which constitutes the main contribution of this paper.

In fact, the idea of introducing nominals to explicitly refer to individual states, can be applied to any logic with a Kripke semantics. Quoting [1], "(..)Strictly speaking, not all modal logics are hybrid, but certainly any modal logics can be hybridized, and in our view many of them should be (...)". This principle is reflected on a recent trend of hybridization of specification formalisms and process calculi. Beyond classical cases of hybrid versions of propositional and first order logic, hybrid accounts of intuitionistic logic [6], $\mathcal{C} \mathcal{T} \mathcal{L}$ [25,20], $\mathcal{L} \mathcal{L}$ [12], $\mu$-calculus [24] among others, are already studied.

What is, thus, in such a context the contribution of this paper? First of all, as stated above, we put forward an institution-independent method to hybridize arbitrary logics, shedding light on the generic pattern of hybridization. In other words, we liberate the essence of hybridization from logical details that are orthogonal to the hybrid idea and that are tributary to other logics.

The hybridization process is also a mechanism for combining logics. Combination of logical system (or institutions), in which typically different roles are played by the different logics to be composed, is, in itself, a relevant research topic. The approach discussed in this paper is in line with the process of modalization of an institution, proposed in [16], in which a modal logic is combined with an arbitrary institution in a systematic way. We take a further step by replacing modal by hybrid logic and allowing multi-modalities.

The paper's second contribution is also a general result: it is shown that any encoding (expressed as 'comorphism' in the sense of [18]) from an arbitrary institution to first order logic $(\mathcal{F O} \mathcal{L})$ determines a comorphism from its (quantifier-free) hybridization to $\mathcal{F} \mathcal{O L}$. Moreover, the proof is constructive entailing a method to define such comorphisms. This may be regarded as a first step for a general theory of encodings of hybrid logics into $\mathcal{F O} \mathcal{L}$ as support for borrowing formal verification tools from $\mathcal{F} \mathcal{O L}$ based to hybrid based specification languages.

Outline. In order to keep exposition reasonably self-contained, Section 2 reviews basic concepts on institutions and recalls a number of examples. The paper's contributions appear on Sections 3 and 4. The former introduces the hybridization process. The latter addresses the construction of comorphisms from hybrid institutions to $\mathcal{F O} \mathcal{L}$. Finally, Section 5 concludes and points out a number of topics for future work. Proofs of all new results presented can be found in the appendix.

\section{Notation and definitions}

Institutions have been defined by Goguen and Burstall in [8], the seminal paper [17] being printed after a delay of many years. Below we recall the concept of institution which formalises the intuitive notion of logical system, including syntax, semantics, and the satisfaction between them. 
Definition 1 (Institution). An institution $\left(\operatorname{Sign}^{\mathcal{I}}, \operatorname{Sen}^{\mathcal{I}}, \operatorname{Mod}^{\mathcal{I}},\left(\models_{\Sigma}^{\mathcal{I}}\right)_{\Sigma \in\left|\operatorname{Sign}^{\mathcal{I}}\right|}\right)$ consists of

- a category $\operatorname{Sign}^{\mathcal{I}}$ whose objects are called signatures,

- a functor $\operatorname{Sen}^{\mathcal{I}}: \operatorname{Sign}^{\mathcal{I}} \rightarrow$ Set giving for each signature a set whose elements are called sentences over that signature,

- a functor $\operatorname{Mod}^{\mathcal{I}}:\left(\operatorname{Sen}^{\mathcal{I}}\right)^{o p} \rightarrow \mathbf{C A T}$, giving for each signature $\Sigma$ a category whose objects are called $\Sigma$-models, and whose arrows are called $\Sigma$-(model) morphisms, and

- a relation $\models{ }_{\Sigma}^{\mathcal{I}} \subseteq\left|\operatorname{Mod}^{\mathcal{I}}(\Sigma)\right| \times \operatorname{Sen}^{\mathcal{I}}$ for each $\Sigma \in\left|\operatorname{Sen}^{\mathcal{I}}\right|$, called the satisfaction relation,

such that for each morphism $\varphi: \Sigma \rightarrow \Sigma^{\prime} \in \operatorname{Sign}^{\mathcal{I}}$, the satisfaction condition

$$
M^{\prime} \models \Sigma^{\mathcal{I}} \operatorname{Sen}^{\mathcal{I}}(\varphi)(\rho) \text { iff } \operatorname{Mod}^{\mathcal{I}}(\varphi)\left(M^{\prime}\right) \models_{\Sigma}^{\mathcal{I}} \rho
$$

holds for each $M^{\prime} \in\left|\operatorname{Mod}^{\mathcal{I}}\left(\Sigma^{\prime}\right)\right|$ and $\rho \in \operatorname{Sen}^{\mathcal{I}}(\Sigma)$.

We recall the notions of amalgamation and quantification space that are crucial for what follows. The former is intensly used in institution theory, whereas the latter was introduced rather recently in [15].

Definition 2 (Amalgamation property). Given any functor Mod : $\operatorname{Sign}^{o p} \rightarrow$ CAT a commuting square of signature morphisms

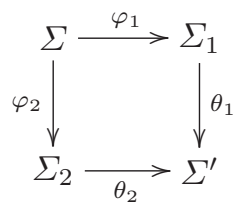

is a weak amalgamation square for Mod if and only if, for each $\Sigma_{1}$-model $M_{1}$ and a $\Sigma_{2}$-model $M_{2}$ such that $\operatorname{Mod}\left(\varphi_{1}\right)\left(M_{1}\right)=\operatorname{Mod}\left(\varphi_{2}\right)\left(M_{2}\right)$, there exists a $\Sigma^{\prime}$ model $M^{\prime}$ such that $\operatorname{Mod}\left(\theta_{1}\right)\left(M^{\prime}\right)=M_{1}$ and $\operatorname{Mod}\left(\theta_{2}\right)\left(M^{\prime}\right)=M_{2}$. When $M^{\prime}$ is required to be unique, the square is called amalgamation square. The model $M^{\prime}$ is called an amalgamation of $M_{1}$ and $M_{2}$ and when it is unique it is denoted by $M_{1} \otimes_{\varphi_{1}, \varphi_{2}} M_{2}$.

When Mod is the model functor $\operatorname{Mod}^{\mathcal{I}}$ of an institution $\mathcal{I}$ we say that $\mathcal{I}$ has the respective amalgamation properties.

Definition 3 (Quantification space). For any category Sign a subclass of arrows $\mathcal{D} \subseteq$ Sign is called a quantification space if, for any $\left(\chi: \Sigma \rightarrow \Sigma^{\prime}\right) \in \mathcal{D}$ and $\varphi: \Sigma \rightarrow \Sigma_{1}$, there is a designated pushout

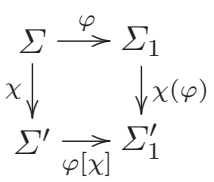


with $\chi(\varphi) \in \mathcal{D}$ and such that the 'horizontal' composition of such designated pushouts is again a designated pushout, i.e. for the pushouts in the following diagram

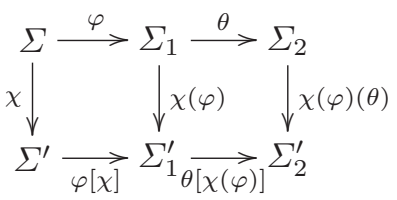

$\varphi[\chi] ; \theta[\chi(\varphi)]=(\varphi ; \theta)[\chi]$ and $\chi(\varphi)(\theta)=\chi(\varphi ; \theta)$, and such that $\chi\left(1_{\Sigma}\right)=\chi$ and $1_{\Sigma}[\chi]=1_{\Sigma}$.

We say that a quantification space $\mathcal{D}$ for $\operatorname{Sign}$ is adequate for a functor Mod : $\operatorname{Sign}^{o p} \rightarrow$ CAT when the designated pushouts mentioned above are weak amalgamation squares for Mod.

Example $1(\mathcal{F} \mathcal{O} \mathcal{L}, \mathcal{A L G}, \mathcal{E} \mathcal{Q}, \mathcal{R} \mathcal{E} \mathcal{L}$ and $\mathcal{P} \mathcal{L})$. A well known example of an institution is $\mathcal{F O} \mathcal{L}$ - the institution of first order logic $\mathcal{F O} \mathcal{L}$ (see [14] for a detailed account). Signatures are tuples $(S, F, P)$ where, $S$ is a set of sort symbols, $F=$ $\left\{F_{w \rightarrow s} \mid s \in S^{*}, s \in S\right\}$ is a family of sets of operation symbols and $P=\left\{P_{w} \mid w \in\right.$ $\left.S^{*}\right\}$ is a family of sets of relational symbols. A signature morphism $\varphi$ is a triple of functions $\left(\varphi_{\text {sort }}, \varphi_{\text {ops }}, \varphi_{\text {pred }}\right):\langle S, F, P\rangle \rightarrow\left\langle S^{\prime}, F^{\prime}, P^{\prime}\right\rangle$, that preserves functionalities, i.e., for any $f \in F_{s_{1} \ldots s_{n} \rightarrow s}, \varphi_{\text {ops }}(f) \in F_{\varphi_{\text {sort }}\left(s_{1}\right) \ldots \varphi_{\text {sort }}\left(s_{n}\right) \rightarrow \varphi_{\text {sort }}(s)}^{\prime}$ and for any $\pi \in P_{s_{1}, \ldots s_{n}}, \varphi_{\text {pred }}(\pi) \in P_{\varphi_{\text {sort }}\left(s_{1}\right) \ldots \varphi_{\text {sort }}\left(s_{n}\right)}^{\prime} \mathrm{A}(S, F, P)$-model $M$ is defined as follows: for each $s \in S, M_{s}$ is a set; for each $f \in F_{s_{1}, \ldots, s_{n} \rightarrow s}, s_{1} \ldots s_{n} \in S^{*}, s \in$ $S, f^{M}: M_{s_{1}} \ldots M_{s_{n}} \rightarrow s$ is a function; and for any $\pi \in P_{s_{1} \ldots s_{n}}, s_{1} \ldots s_{n} \in S^{*}$, $\pi^{M} \subseteq M_{s_{1}} \times \cdots \times M_{s_{n}}$. $(S, F, P)$-models homomorphisms are $S$-families of functions $\left\{h_{s}: M_{s} \rightarrow M_{s}^{\prime}\right\}_{s \in S}$, such that for any $f \in F_{s_{1} \ldots s_{n} \rightarrow s}$, and each $m_{i} \in M_{s_{i}}$, $i=1, \ldots, n, h_{s}\left(f^{M}\left(m_{1}, \ldots, m_{n}\right)\right)=f^{M^{\prime}}\left(h_{s}\left(m_{1}\right), \ldots, h_{s}\left(m_{1}\right)\right)$ and for each $\pi \in P_{s_{1}, \ldots s_{n}}$, if $\left(m_{1}, \ldots, m_{n}\right) \in \pi^{M}$ then $\left(h_{s}\left(m_{1}\right), \ldots, h_{s}\left(m_{n}\right)\right) \in \pi^{M^{\prime}}$. A reduct of a $\left(S^{\prime}, F^{\prime}, P^{\prime}\right)$-model $M^{\prime}$ along $\varphi$ consists of the $(S, F, P)$-model $M^{\prime} \uparrow_{\varphi}$ defined for each $s \in S$ by $\left(M^{\prime} \uparrow_{\varphi}\right)_{s}=M_{s}^{\prime}$, for each $f \in F_{s_{1}, \ldots, s_{n}}, f^{M^{\prime} \uparrow \varphi}=\varphi_{\text {ops }}(f)^{M^{\prime}}$ and for each $\pi \in P_{s_{1} \ldots s_{n}}, \varphi^{M^{\prime} \uparrow_{\varphi}}=\varphi_{\text {pred }}(\pi)^{M^{\prime}}$.

The set $\operatorname{Sen}^{\mathcal{F} \mathcal{O} \mathcal{L}}(\langle S, F, P\rangle)$ of $(S, F, P)$-sentences consists of the usual firstorder $(S, F, P)$-formulas, i.e., the smallest set which contains all $(S, F)$-equations (formally, $t \approx t^{\prime}$ is in $\operatorname{Sen}^{\mathcal{F O} \mathcal{L}}\left(\langle S, F, P\rangle\right.$ ), for each $t, t^{\prime} \in\left(T_{(S, F)}\right)_{s}, s \in S$ ), predicate terms $\left(\pi\left(t_{1}, \ldots, t_{n}\right)\right.$, for any $\left.\pi \in P_{s_{1} \ldots s_{n}}, t_{i} \in\left(T_{(S, F)}\right)_{s_{i}}\right)$ and their closure by application of the usual boolean connectives $\left(\neg \rho\right.$ and $\rho \odot \rho^{\prime}$, for any $\rho, \rho^{\prime} \in \operatorname{Sen}^{\mathcal{F} \mathcal{O L}}(\langle S, F, P\rangle), \odot \in\{\vee, \wedge, \rightarrow\}$ ) and quantifiers (for any $\rho \in$ $\operatorname{Sen}^{\mathcal{F} \mathcal{O} \mathcal{L}}(\langle S, F \uplus X, P\rangle), \forall X \rho$ and $\exists X \rho$ are in $\left.\operatorname{Sen}^{\mathcal{F O} \mathcal{L}}(\langle S, F, P\rangle)\right)$. A signature morphism $\varphi:\langle S, F, P\rangle \rightarrow\left\langle S^{\prime}, F^{\prime}, P^{\prime}\right\rangle$ induces a translation of sentences, $\operatorname{Sen}^{\mathcal{F O} \mathcal{L}}(\varphi): \operatorname{Sen}^{\mathcal{F} \mathcal{O L}}(\langle S, F, P\rangle) \rightarrow \operatorname{Sen}^{\mathcal{F} \mathcal{O} \mathcal{L}}\left(\left\langle S^{\prime}, F^{\prime}, P^{\prime}\right\rangle\right)$, that replaces symbols of $\langle S, F, P\rangle$ by the respective $\varphi$-images in $\left\langle S^{\prime}, F^{\prime}, P^{\prime}\right\rangle$. More precisely, let function $\varphi^{t r m}: T_{(S, F)} \rightarrow T_{\left(S^{\prime}, F^{\prime}\right)}$ be defined by

$$
\varphi^{t r m}\left(f\left(t_{1}, \ldots, t_{n}\right)\right)=\varphi_{o p s}(f)\left(\varphi^{t r m}\left(t_{1}\right), \ldots, \varphi^{t r m}\left(t_{n}\right)\right)
$$

Then, translation is recursively defined as follows: 
$-\operatorname{Sen}^{\mathcal{F} \mathcal{O L}}(\varphi)\left(t \approx t^{\prime}\right)=\varphi^{t r m}(t) \approx \varphi^{t r m}\left(t^{\prime}\right)$

$\left.-\operatorname{Sen}^{\mathcal{F O} \mathcal{L}}(\varphi)\left(\pi\left(t_{1}\right), \ldots, \pi\left(t_{n}\right)\right)=\varphi_{\text {pred }}(\pi)\left(\varphi^{\text {trm }}\left(t_{1}\right), \ldots, \varphi^{\text {trm }}\left(t_{n}\right)\right)\right) ;$

$-\operatorname{Sen}^{\mathcal{F O L}}(\varphi)\left(\neg \rho^{\prime}\right)=\neg \operatorname{Sen}^{\mathcal{F O} \mathcal{L}}(\varphi)\left(\rho^{\prime}\right)$

$-\operatorname{Sen}^{\mathcal{F O L}}(\varphi)\left(\rho \odot \rho^{\prime}\right)=\operatorname{Sen}^{\mathcal{F O} \mathcal{L}}(\varphi)(\rho) \odot \operatorname{Sen}^{\mathcal{F O L}}(\varphi)\left(\rho^{\prime}\right), \odot \in\{\vee, \wedge, \rightarrow\} ;$

- $\operatorname{Sen}^{\mathcal{F O} \mathcal{L}}(\varphi)(\forall X \rho)=\forall X^{\varphi} \operatorname{Sen}^{\mathcal{F O} \mathcal{L}}\left(\varphi^{\prime}\right)(\rho)$, where

$X^{\varphi}=\left\{\left(x, \varphi_{s t}(s),\left(S^{\prime}, F^{\prime}, P^{\prime}\right)\right) \mid(x, s,(S, F, P))\right\}$, and $\varphi^{\prime}$ canonically extends $\varphi$ by mapping each $(x, s,(S, F, P))$ to $\left(x, \varphi_{s t}(s),\left(S^{\prime}, F^{\prime}, P^{\prime}\right)\right)$.

Finally, satisfaction is given by the Tarskian satisfaction relation recursively defined as follows:

$-\mathcal{M} \models{ }_{\langle S, F, P\rangle}^{\mathcal{F O} \mathcal{L}} t \approx t^{\prime}$ if $t^{M}=t^{\prime M} ;$

$-\mathcal{M} \models \underset{\langle S, F, P\rangle}{\mathcal{F} \mathcal{O} \mathcal{L}} \pi\left(t_{1}, \ldots, t_{n}\right)$ if $\left(t_{1}^{M}, \ldots, t_{n}^{M}\right) \in \pi^{M}$;

$-\mathcal{M} \models_{\langle S, F, P\rangle}^{\mathcal{F} \mathcal{O} \mathcal{L}} \rho \vee \rho^{\prime}$ if $\mathcal{M} \models_{\langle S, F, P\rangle}^{\mathcal{F O} \mathcal{L}} \rho$ or $\mathcal{M} \models \rho^{\prime}$ and similarly for the remaining boolean connectives;

- $\mathcal{M} \models_{\langle S, F, P\rangle}^{\mathcal{F} \mathcal{O}} \forall X \rho$ if $\mathcal{M}^{\prime} \models_{\langle S, F \uplus X, P\rangle}^{\mathcal{F} \mathcal{L}} \rho$, for each expansion $M^{\prime}$ of $M$ along the signature morphism $(S, F, P) \hookrightarrow(S, F \uplus X, P)$;

$-\mathcal{M} \models \underset{\langle S, F, P\rangle}{\mathcal{F} \mathcal{O} \mathcal{L}} \exists X \rho$ if $\mathcal{M} \models \underset{\langle S, F, P\rangle}{\mathcal{F} \mathcal{O L}} \neg \forall X \neg \rho$

The institution $\mathcal{A L G}$ is obtained by $\mathcal{F O} \mathcal{L}$ by discarding the relational symbols and the corresponding interpretations in models. The institution $\mathcal{E} \mathcal{Q}$ is defined as the sub-institution of $\mathcal{A L G}$ where the sentences are just universally quantified equations $(\forall X) t \approx t^{\prime}$. The institution $\mathcal{R E} \mathcal{L}$ is the sub-institution of single-sorted first-order logic with signatures having only constants and relational symbols.

The institution $\mathcal{P L}$ (of propositional logic) is the fragment of $\mathcal{F O} \mathcal{L}$ determined by signatures with empty sets of sort symbols.

\section{A method to hybridize arbitrary institutions}

Let us consider an institution $\mathcal{I}=\left(\operatorname{Sign}^{\mathcal{I}}, \operatorname{Sen}^{\mathcal{I}}, \operatorname{Mod}^{\mathcal{I}},\left(\models_{\Sigma}^{\mathcal{I}}\right)_{\Sigma \in\left|\operatorname{Sign}^{\mathcal{I}}\right|}\right)$ with a designated quantification space $\mathcal{D}^{\mathcal{I}} \subseteq$ Sign. This section introduces a method to enrich the expressivity of $\mathcal{I}$ with modalities and nominals, defining a suitable semantics for it. Moreover, it is shown that the outcome still defines an institution, to which we refer as the hybrid $\mathcal{I}$ and denote by $\mathcal{H} \mathcal{I}$.

\section{The category of $\mathcal{H I}$-signatures}

The category of $\mathcal{I}$-hybrid signatures, denoted by $\operatorname{Sign}^{\mathcal{H I}}$, is defined as the following direct (cartesian) product of categories:

$$
\operatorname{Sign}^{\mathcal{H} \mathcal{I}}=\operatorname{Sign}^{\mathcal{I}} \times \operatorname{Sign}^{\mathcal{R E} \mathcal{L}} .
$$

The $\mathcal{R E} \mathcal{L}$-signatures are denoted by (Nom, $\Lambda$ ), where Nom is a set of constants called nominals and $\Lambda$ is a set of relational symbols called modalities; $\Lambda_{n}$ stands for the set of modalities of arity $n$. General category theory entails, 
Proposition 1. The projection $\operatorname{Sign}^{\mathcal{H I}} \rightarrow \operatorname{Sign}^{\mathcal{I}}$ lifts small co-limits.

The existence of co-limits of signatures is one of the properties of institutions of key practical relevance for specification in-the-large (see [17]).

Corollary 1. Sign ${ }^{\mathcal{H I}}$ has all small co-limits.

\section{$\mathcal{H} \mathcal{I}$-sentences}

Let us fix a quantification space $\mathcal{D}^{\mathcal{H I}}$ for $\operatorname{Sign}{ }^{\mathcal{H I}}$ such that for each $\chi \in \mathcal{D}^{\mathcal{H I}}$ its projection $\left.\chi\right|_{\mathcal{I}}$ to $\operatorname{Sign}^{\mathcal{I}}$ belongs to $\mathcal{D}^{\mathcal{I}}$. The quantification space $\mathcal{D}^{\mathcal{H} \mathcal{I}}$ is a parameter of the hybridization process. Whenever $\mathcal{D}^{\mathcal{H I}}$ consists of identities we say the hybridization is quantifier-free. Note that a quantifier-free hybridization does not necessarily mean the absence of 'local' quantification, i.e. placed at the level of base institution $\mathcal{I}$.

Let $\Delta=(\Sigma, \operatorname{Nom}, \Lambda)$. The set of sentences $\operatorname{Sen}^{\mathcal{H I}}(\Delta)$ is the least set such that

$-\operatorname{Nom} \subseteq \operatorname{Sen}^{\mathcal{H I}}(\Delta)$

$-\operatorname{Sen}^{\mathcal{I}}(\Sigma) \subseteq \operatorname{Sen}^{\mathcal{H} \mathcal{I}}(\Delta)$

$-\rho \odot \rho^{\prime} \in \operatorname{Sen}^{\mathcal{H I}}(\Delta)$ for any $\rho, \rho^{\prime} \in \operatorname{Sen}^{\mathcal{H I}}(\Delta)$ and any $\odot \in\{\vee, \wedge, \rightarrow\}$,

- $\neg \rho \in \operatorname{Sen}^{\mathcal{H I}}(\Delta)$, for any $\rho \in \operatorname{Sen}^{\mathcal{H I}}(\Delta)$,

- $@_{i} \rho \in \operatorname{Sen}^{\mathcal{H I}}(\Delta)$ for any $\rho \in \operatorname{Sen}(\Sigma)$ and $i \in$ Nom;

- $[\lambda]\left(\rho_{1}, \ldots, \rho_{n}\right) \in \operatorname{Sen}^{\mathcal{H I}}(\Delta)$, for any $\lambda \in \Lambda_{n+1}, \rho_{i} \in \operatorname{Sen}^{\mathcal{H I}}(\Delta), i \in\{1, \ldots, n\}$;

- $(\forall \chi) \rho \in \operatorname{Sen}^{\mathcal{H I}}(\Delta)$, for any $\rho \in \operatorname{Sen}^{\mathcal{H I}}\left(\Delta^{\prime}\right)$ and $\chi: \Delta \rightarrow \Delta^{\prime} \in \mathcal{D}^{\mathcal{H I}}$;

- $(\exists \chi) \rho \in \operatorname{Sen}^{\mathcal{H I}}(\Delta)$, for any $\rho \in \operatorname{Sen}^{\mathcal{H I}}\left(\Delta^{\prime}\right)$ and $\chi: \Delta \rightarrow \Delta^{\prime} \in \mathcal{D}^{\mathcal{H I}}$;

\section{Translations of $\mathcal{H} \mathcal{I}$-sentences}

Let $\varphi=\left(\varphi_{\mathrm{Sig}}, \varphi_{\mathrm{Nom}}, \varphi_{\mathrm{MS}}\right):(\Sigma, \operatorname{Nom}, \Lambda) \rightarrow\left(\Sigma^{\prime}, \mathrm{Nom}^{\prime}, \Lambda^{\prime}\right)$ be a morphims of $\mathcal{H} \mathcal{I}$-signatures.

The translation $\operatorname{Sen}^{\mathcal{H I}}(\varphi)$ is defined as follows:

- $\operatorname{Sen}^{\mathcal{H I}}(\varphi)(\rho)=\operatorname{Sen}^{\mathcal{I}}\left(\varphi_{\mathrm{Sig}}\right)(\rho)$ for any $\rho \in \operatorname{Sen}^{\mathcal{I}}(\Sigma)$;

$-\operatorname{Sen}^{\mathcal{H I}}(\varphi)(i)=\varphi_{\text {Nom }}(i)$

- $\operatorname{Sen}^{\mathcal{H I}}(\varphi)(\neg \rho)=\neg \operatorname{Sen}^{\mathcal{H I}}(\varphi)(\rho)$;

- $\operatorname{Sen}^{\mathcal{H I}}(\varphi)\left(\rho \odot \rho^{\prime}\right)=\operatorname{Sen}^{\mathcal{H I}}(\varphi)(\rho) \odot \operatorname{Sen}^{\mathcal{H I}}(\varphi)\left(\rho^{\prime}\right), \odot \in\{\vee, \wedge, \rightarrow\} ;$

$-\operatorname{Sen}^{\mathcal{H I}}(\varphi)\left(@_{i} \rho\right)=@_{\varphi_{\mathrm{Nom}}(i)} \operatorname{Sen}^{\mathcal{H I}}(\rho)$;

$-\operatorname{Sen}^{\mathcal{H I}}(\varphi)\left([\lambda]\left(\rho_{1}, \ldots, \rho_{n}\right)\right)=\left[\varphi_{\mathrm{MS}}(\lambda)\right]\left(\operatorname{Sen}^{\mathcal{H I}}\left(\rho_{1}\right), \ldots, \operatorname{Sen}^{\mathcal{H I}}\left(\rho_{n}\right)\right) ;$

- $\operatorname{Sen}^{\mathcal{H I}}(\varphi)((\forall \chi) \rho)=(\forall \chi(\varphi)) \operatorname{Sen}^{\mathcal{H I}}(\varphi[\chi])(\rho)$.

Proposition 2. $\operatorname{Sen}^{\mathcal{H I}}$ is a functor $\operatorname{Sign}^{\mathcal{H} I} \rightarrow$ Set. 


\section{$\mathcal{H} \mathcal{I}$-models}

The $(\Sigma$, Nom, $\Lambda)$-models are pairs

$$
\mathcal{M}=(M, R) \text { where }
$$

- $R$ is a (Nom, $\Lambda$ )-model in $\mathcal{R E} \mathcal{L}$;

$-M$ is a function $|R| \rightarrow\left|\operatorname{Mod}^{\mathcal{I}}(\Sigma)\right|$.

The carrier set $|R|$ forms the set of the states of $\mathcal{M} ;\left\{n^{R} \mid n \in\right.$ Nom $\}$ represents the interpretations of the nominals Nom, whereas relations $\left\{\lambda^{R} \mid \lambda \in \Lambda_{n}, n \in \omega\right\}$ represent the interpretation of the modalities $\Lambda$. We denote $M(s)$ simply by $M_{s}$.

A (Nom, $\Lambda)$-model homomorphism $h:(M, R) \rightarrow\left(M^{\prime}, R^{\prime}\right)$ consists of a pair aggregating

- a (Nom, $\Lambda$ )-model homomorphism in $\mathcal{R} \mathcal{E} \mathcal{L}, h_{s t}: R \rightarrow R^{\prime}$; i.e., a function $h:|R| \rightarrow\left|R^{\prime}\right|$ such that for $i \in$ Nom, $i^{R^{\prime}}=h_{s t}\left(i^{R}\right)$; and, for any $s_{1}, \ldots, s_{n} \in$ $|R|$, and $\lambda \in \Lambda_{n},\left(s_{1}, \ldots, s_{n}\right) \in \lambda^{R},\left(h_{s t}\left(s_{1}\right), \ldots, h_{s t}\left(s_{n}\right)\right) \in \lambda^{R^{\prime}}$.

- a natural transformation $h_{\text {mod }}: M \Rightarrow M^{\prime} \circ h_{s t}$; note that $h_{\text {mod }}$ is a $|R|$ indexed family of $\Sigma$-model homomorphisms $h_{\text {mod }}=\left\{h_{\text {mod }}^{s}: M_{s} \rightarrow M_{h_{s t}(s)}^{\prime}\right\}_{s \in|R|}$.

The composition of hybrid model homomorphisms is defined canonically as

$$
h ; h^{\prime}=\left(h_{s t} ; h_{s t}^{\prime}, h_{m o d} ;\left(h_{m o d}^{\prime} \circ h_{s t}\right)\right) .
$$

Fact 1 Let $\Delta$ be any hybrid signature over an institution $\mathcal{I}$. Then $\Delta$-models together with their homomorphisms constitute a category.

\section{Reducts of $\mathcal{H} \mathcal{I}$-models}

Let $\Delta=(\Sigma$, Nom, $\Lambda)$ and $\Delta^{\prime}=\left(\Sigma^{\prime}\right.$, Nom $\left.^{\prime}, \Lambda^{\prime}\right)$ be two hybrid signatures, $\varphi=$ $\left(\varphi_{\mathrm{Sig}}, \varphi_{\mathrm{Nom}}, \varphi_{\mathrm{MS}}\right)$ a morphism between $\Delta$ and $\Delta^{\prime}$ and $\left(M^{\prime}, R^{\prime}\right)$ a $\Delta^{\prime}$-model. The reduct of $\left(M^{\prime}, R^{\prime}\right)$ along $\varphi$, denoted by $\operatorname{Mod}^{\mathcal{H I}}(\varphi)\left(M^{\prime}, R^{\prime}\right)$, is the $\Delta$-model $(M, R)$ such that

$-|R|=\left|R^{\prime}\right|$

- for any $n \in$ Nom, $n^{R}=\varphi_{\text {Nom }}(n)^{R^{\prime}}$;

- for any $\lambda \in \Lambda, \lambda^{R}=\varphi_{\mathrm{MS}}(\lambda)^{R^{\prime}}$;

- for any $s \in|R|, M_{s}=\operatorname{Mod}^{\mathcal{I}}\left(\varphi_{\mathrm{Sig}}\right)\left(M_{s}^{\prime}\right)$.

Theorem 1. A pushout square of $\mathcal{H} \mathcal{I}$-signature morphisms is a (weak) amalgamation square (for $\operatorname{Mod}^{\mathcal{H} \mathcal{I}}$ ) if the underlying square of signature morphisms in $\mathcal{I}$ is a (weak) amalgamation square.

Corollary 2. $\mathcal{D}^{\mathcal{H I}}$ is adequate for $\operatorname{Mod}^{\mathcal{H I}}$. 


\section{The Satisfaction relation}

For any $(\Sigma$, Nom, $\Lambda)$-model $(M, R)$ and for any $s \in|R|$ :

- $(M, R) \models^{s} \rho$ iff $M_{s} \models^{\mathcal{I}} \rho$; when $\rho \in \operatorname{Sen}^{\mathcal{I}}(\Sigma)$,

- $(M, R) \models^{s} i$ iff $i^{R}=s$; when $i \in$ Nom,

- $(M, R) \models^{s} \rho \vee \rho^{\prime}$ iff $(M, R) \models{ }^{s} \rho$ or $(M, R) \models{ }^{s} \rho^{\prime}$,

$-(M, R) \models{ }^{s} \rho \wedge \rho^{\prime}$ iff $(M, R) \models{ }^{s} \rho$ and $(M, R) \models{ }^{s} \rho^{\prime}$,

- $(M, R) \models{ }^{s} \rho \rightarrow \rho^{\prime}$ iff $(M, R) \models{ }^{s} \rho$ implies that $(M, R) \models{ }^{s} \rho^{\prime}$,

$-(M, R) \models^{s}[\lambda]\left(\rho_{1}, \ldots \rho_{n}\right)$ iff $(M, R) \models^{s_{i}} \rho_{i}$ for $1 \leq i \leq n$ for any $\left(s, s_{1}, \ldots, s_{n}\right) \in$ $R_{\lambda}, \lambda \in \Lambda_{n+1}$,

$-(M, R) \models{ }^{s} @_{j} \rho$ iff $(M, R) \models^{j^{R}} \rho$,

- $(M, R) \models^{s}(\forall \chi) \rho$ iff $\left(M^{\prime}, R^{\prime}\right) \models^{s} \rho$ for any $\left(M^{\prime}, R^{\prime}\right)$ such that $\operatorname{Mod}^{\mathcal{H I}}(\chi)\left(M^{\prime}, R^{\prime}\right)=$ $(M, R)$, and

- $(M, R) \models^{s}(\exists \chi) \rho$ iff $\left(M^{\prime}, R^{\prime}\right) \models^{s} \rho$ for some $\left(M^{\prime}, R^{\prime}\right)$ such that $\operatorname{Mod}^{\mathcal{H I}}(\chi)\left(M^{\prime}, R^{\prime}\right)=$ $(M, R)$.

We write $(M, R) \models \rho$ iff $(M, R) \models^{s} \rho$ for any $s \in|R|$.

\section{The Satisfaction Condition}

Theorem 2. Let $\Delta=(\Sigma, \operatorname{Nom}, \Lambda)$ and $\Delta^{\prime}=\left(\Sigma^{\prime}, \mathrm{Nom}^{\prime}, \Lambda^{\prime}\right)$ be two $\mathcal{H} \mathcal{I}$-signatures and $\varphi: \Delta \rightarrow \Delta^{\prime}$ a morphism of signatures. For any $\rho \in \operatorname{Sen}^{\mathcal{H} \mathcal{I}}(\Delta),\left(M^{\prime}, R^{\prime}\right) \in$ $\left|\operatorname{Mod}^{\mathcal{H I}}\left(\Delta^{\prime}\right)\right|$, and $s \in|R|$

$$
\operatorname{Mod}^{\mathcal{H I}}(\varphi)\left(M^{\prime}, R^{\prime}\right) \models^{s} \rho \text { iff }\left(M^{\prime}, R^{\prime}\right) \models{ }^{s} \operatorname{Sen}^{\mathcal{H I}}(\varphi)(\rho) .
$$

Corollary 3 (The Satisfaction Condition). $\left(\operatorname{Sign}^{\mathcal{H I}}, \operatorname{Sen}^{\mathcal{H I}}, \operatorname{Mod}^{\mathcal{H} \mathcal{I}}, \models^{\mathcal{H I}}\right)$ is an institution.

Example 2 $(\mathcal{H} \mathcal{P} \mathcal{L})$. Let $\mathcal{A P} \mathcal{L}$ be the sub-institution of $\mathcal{P} \mathcal{L}$ whose sentences are the propositional symbols. Applying the hybridization method described above to $\mathcal{A P} \mathcal{L}$ and fixing $\Lambda_{2}=\{\square\}$ and $\Lambda_{n}=\emptyset$ for each $n \neq 2$, we obtain the institution of the "standard" hybrid propositional logic (without state quantifiers): the category of signatures is $\operatorname{Sign}^{\mathcal{H P} \mathcal{L}}=$ Set $\times$ Set with objects denoted by $(P$, Nom $)$ and morphisms by $\left(\varphi_{\mathrm{Sig}}, \varphi_{\text {Nom }}\right)$; sentences are the usual hybrid propositional formulas, i.e., modal formulas closed by boolean connectives, $\square$, and by the operator $@_{i}, i \in$ Nom; models consists of pairs $\mathcal{P}=(M, R)$ where $R$ consists of a carrier set, interpretations $i^{R} \in S$ for each $i \in$ Nom, and a binary relation $\square^{R} \subseteq|R| \times|R|$, and, for each $s \in|R|, M_{s}$ is a propositional model, i.e., a function $M_{s}: P \rightarrow\{\top, \perp\}$. The quantification space $\mathcal{D}^{\mathcal{H P} \mathcal{L}}$ is the trivial one, consisting of the identities, which means this process is a quantifier-free hybridization. The satisfaction relation is defined as above on top of the propositional satisfaction relation, i.e., $\mathcal{P} \models{ }^{s} p$ iff $M_{s}(p)=\top$.

A challenging issue concerns finding suitable quantification spaces to capture other versions of hybrid propositional logic. For instance, it would be interesting, 
along the hybridization process, to capture quantifiers $\mathbf{A}$ and $\mathbf{E}$, where $\mathbf{A} \rho$ (respectively, $\mathbf{E} \rho$ ) means that " $\rho$ is true in all the states of the model" (respectively, " $\rho$ is true in some state of the model") [1]. Considering as a quantification space the extensions of signatures with nominal symbols, paves the way to express the following properties:

\begin{tabular}{|l|l|c|}
\hline $\mathcal{P} \models$ & iff $((\forall i) i) \leftrightarrow \rho$ & $\rho$ is satisfied at $s$ iff $s$ is unique in $\mathcal{P}$ \\
\hline $\mathcal{P} \models \models^{s}(\forall i) @_{i} \rho$ & iff & $\mathcal{P} \models \rho$ \\
\hline $\mathcal{P} \models{ }^{s}(\exists i) @_{i} \rho$ & iff & $\mathcal{P} \models \mathbf{E} \rho$ \\
\hline
\end{tabular}

Example $3(\mathcal{H} \mathcal{F} \mathcal{O} \mathcal{L}, \mathcal{H} \mathcal{E})$. The application of the hybridization method to $\mathcal{F O} \mathcal{L}$ taking as a quantification space signature extensions both with $\mathcal{F O} \mathcal{L}$ variables and variables over nominals, one captures the state-variables quantification of first-order hybrid logic of [7].

Binding "state variables" to the point of evaluation highly increase the expressive power of an hybrid logic, which is enabled through the binder operator $\downarrow$ (e.g. $[2,7])$. This may be achieved by taking $i$-expansions $\chi:(\Sigma$,Nom, $\Lambda) \hookrightarrow$ $(\Sigma$, Nom $\uplus\{i\}, \Lambda)$ as a quantification space and including, on defining satisfaction, the condition

$-\mathcal{P} \models^{s}(\downarrow \chi) \rho$ iff for any $\chi$-expansion $\mathcal{P}^{\prime}$ of $\mathcal{P}$ such that $i^{R}=s$, we have $\mathcal{P}^{\prime} \models{ }^{s} \rho$.

As a final example, let us mention the hybridization of $\mathcal{E} \mathcal{Q}$ with the trivial quantification space. The resulting hybrid equational institution provides a suitable setting for specifying evolving systems in which each state is endowed with an specific algebra [21].

\section{$4 \mathcal{F} \mathcal{O} \mathcal{L}$ as a support to hybrid specification}

This section studies the existence of encodings of hybrid institutions into $\mathcal{F O} \mathcal{L}$. The relevance of such encodings is to provide proof theoretic support to hybrid specifications. In particular, we show that any encoding of the base institution $\mathcal{I}$ to $\mathcal{F O} \mathcal{L}$ may be lifted to an encoding of the quantifier-free hybrid institution $\mathcal{H} \mathcal{I}$ to $\mathcal{F O} \mathcal{L}$. Our approach to logic encodings relies upon the concept of comorphism, recalled below from the literature (e.g. [18]).

Definition 4 (Comorphisms). Given institutions $\mathcal{I}=($ Sign, Sen, Mod, $\models)$ and $\mathcal{I}^{\prime}=\left(\operatorname{Sign}^{\prime}, \operatorname{Sen}^{\prime}, \operatorname{Mod}^{\prime}, \models^{\prime}\right)$ a comorphism $(\Phi, \alpha, \beta): \mathcal{I} \rightarrow \mathcal{I}^{\prime}$ consists of

1. a functor $\Phi:$ Sign $\rightarrow$ Sign', $^{\prime}$

2. a natural transformation $\alpha$ : Sen $\Rightarrow \Phi ;$ Sen $^{\prime}$, and

3. a natural transformation $\beta$ : $\Phi^{o p} ; \operatorname{Mod}^{\prime} \Rightarrow \operatorname{Mod}$

such that the following satisfaction condition holds

$$
M^{\prime} \models_{\Phi(\Sigma)}^{\prime} \alpha_{\Sigma}(e) \text { iff } \beta_{\Sigma}\left(M^{\prime}\right) \models_{\Sigma} e
$$


for each signature $\Sigma \in|\operatorname{Sign}|, \Phi(\Sigma)$-model $M^{\prime}$, and $\Sigma$-sentence $e$.

The comorphism is conservative whenever, for each $\Sigma$-model $M$ in $\mathcal{I}$, there exists a $\Phi(\Sigma)$-model $M^{\prime}$ in $\mathcal{I}^{\prime}$ such that $M=\beta_{\Sigma}\left(M^{\prime}\right)$.

The following is a consequence of conservativity, with the important proof theoretic implication in that we may prove things in the source institution by using the proof system of the target institution in a sound and complete way.

Fact 2 For any set $\Gamma \subseteq \operatorname{Sen}(\Sigma)$ and sentence $\rho \in \operatorname{Sen}(\Sigma)$,

$$
\Gamma \models_{\Sigma} \rho \text { if and only if } \alpha_{\Sigma}(\Gamma) \models_{\Phi(\Sigma)}^{\prime} \alpha_{\Sigma}(\rho) .
$$

Example 4. One may legitimately wonder about the existence of a canonical embedding of the base institution $\mathcal{I}$ into its hybridization $\mathcal{H} \mathcal{I}$ in the form of a comorphism $(\Phi, \alpha, \beta): \mathcal{I} \rightarrow \mathcal{H I}$. The answer is as follows:

$-\Phi(\Sigma)=(\Sigma,\{i\}, \emptyset)$,

$-\alpha_{\Sigma}(\rho)=@_{i} \rho$, and

$-\beta_{\Sigma}(M, R)=M_{i^{R}}$.

It is easy to show that this is a conservative comorphism.

Thus, let $\mathcal{H I}$ be the quantifier-free hybridization of institution $\mathcal{I}$. Given any comorphism $\mathcal{I} \stackrel{(\Phi, \alpha, \beta)}{\longrightarrow} \mathcal{F} \mathcal{O} \mathcal{L}$ we define a comorphism $\mathcal{H} \mathcal{I}_{\left(\Phi^{\prime}, \alpha^{\prime}, \beta^{\prime}\right)}^{\longrightarrow} \mathcal{F} \mathcal{O} \mathcal{L}$ by

Translation of the signatures:

$$
\begin{aligned}
& \Phi^{\prime}(\Sigma, \text { Nom, } \Lambda)=\left(S_{\Sigma}+\{\mathrm{ST}\}, \overline{F_{\Sigma}}+\overline{\mathrm{Nom}}, \overline{P_{\Sigma}}+\bar{\Lambda}\right) \text { where } \\
& -\Phi(\Sigma)=\left(S_{\Sigma}, P_{\Sigma}, P_{\Sigma}\right)(\text { a } \mathcal{F} \mathcal{O} \mathcal{L} \text { signature }) ; \\
& -\overline{F_{\Sigma}}= \begin{cases}\left(\overline{F_{\Sigma}}\right)_{\mathrm{ST} w \rightarrow s}=\left(F_{\Sigma}\right)_{w \rightarrow s} & \text { for any } s \in S_{\Sigma}, w \in S_{\Sigma}^{*} \\
\emptyset, & \text { for the other cases }\end{cases} \\
& -\overline{P_{\Sigma}}= \begin{cases}\left(\overline{P_{\Sigma}}\right)_{\mathrm{ST} w}=\left(P_{\Sigma}\right)_{w} & \text { for any } w \in S_{\Sigma}^{*} ; \\
\emptyset, & \text { for the other cases }\end{cases} \\
& -\overline{\mathrm{Nom}}=\{i: \rightarrow \mathrm{ST} \mid i \in \mathrm{Nom}\} \\
& -\bar{\Lambda}=\left\{\lambda: \mathrm{ST}^{n} \mid \lambda \in \Lambda_{n}\right\} .
\end{aligned}
$$

\section{Translation of the models:}

$\beta_{(\Sigma, \mathrm{Nom}, \Lambda)}^{\prime}(M)=\left(M^{\prime}, R\right)$ where

- $R$ is the reduct $M \uparrow_{(\{\mathrm{ST}\}, \overline{\mathrm{Nom}}, \bar{\Lambda})}$, and

$-M^{\prime}: \mathrm{ST}^{R} \rightarrow\left|\operatorname{Mod}^{\mathcal{I}}(\Sigma)\right|$ is defined for each $s \in S$ by $M_{s}^{\prime}=\beta_{\Sigma}\left(M_{s}\right)$ where $M_{s}$ is the $\Phi(\Sigma)=\left(S_{\Sigma}, P_{\Sigma}, P_{\Sigma}\right)$-model defined by

- for each sort $\in S_{\Sigma}$, $\operatorname{sort}^{M_{s}}=\operatorname{sort}^{M}$;

- for each $f \in F_{\Sigma}, f^{M_{s}}(m)=f^{M}(s, m)$;

- for each $\pi \in P_{\Sigma}, m \in \pi^{M_{s}}$ iff $(s, m) \in \pi^{M}$. 


\section{Translation of the sentences:}

$\alpha_{(\Sigma, \mathrm{Nom}, \Lambda)}^{\prime}(\rho)=\forall x \alpha_{(\Sigma, \mathrm{Nom}, \Lambda)}^{\prime x}(\rho)$, where

$\alpha_{(\Sigma, \mathrm{Nom}, \Lambda)}^{\prime x}: \operatorname{Sen}^{\mathcal{H I}}(\Sigma$, Nom, $\Lambda) \rightarrow \operatorname{Sen}^{\mathcal{F O} \mathcal{L}}\left(\Phi^{\prime}(\Sigma\right.$, Nom, $\left.\Lambda) \cup\{x\}\right)$ with $x$ being a constant of sort ST, is defined by

- for each $\rho \in \operatorname{Sen}^{\mathcal{I}}(\Sigma), \alpha^{\prime x}(\rho)=\alpha^{x}\left(\alpha_{\Sigma}(\rho)\right)$ where $\alpha_{(\Sigma, \mathrm{Nom}, \Lambda)}^{x}: \operatorname{Sen}^{\mathcal{F O} \mathcal{L}}(\Phi(\Sigma)) \rightarrow$ $\operatorname{Sen}^{\mathcal{F O L}}\left(\Phi^{\prime}(\Sigma\right.$, Nom, $\left.\Lambda) \cup\{x\}\right)$ is defined by

- $\alpha^{x}\left(t \approx t^{\prime}\right)=\alpha^{x}(t) \approx \alpha^{x}\left(t^{\prime}\right)$ where $\alpha^{x}\left(f\left(t_{1}, \ldots, t_{n}\right)\right)=f\left(x, \alpha^{x}\left(t_{1}\right), \ldots, \alpha^{x}\left(t_{n}\right)\right)$;

- $\alpha^{x}(\pi(t))=\pi\left(x, \alpha^{x}(t)\right)$

- $\alpha^{x}\left(\rho_{1} \odot \rho_{2}\right)=\alpha^{x}\left(\rho_{1}\right) \odot \alpha^{x}\left(\rho_{2}\right), \odot \in\{\vee, \wedge, \rightarrow\}$

- $\alpha^{x}(\neg \rho)=\neg \alpha^{x}(\rho)$;

- $\alpha^{x}(\forall y \rho)=\forall y \alpha^{x}(\rho)$

$-\alpha^{\prime x}(i)=i \approx x, i \in$ Nom;

$-\alpha^{\prime x}\left(@_{i} \rho\right)=\alpha^{\prime i}(\rho)$

- $\alpha^{\prime x}\left([\lambda]\left(\rho_{1}, \ldots, \rho_{n}\right)\right)=\forall y_{1}, \ldots, y_{n}\left(\lambda\left(x, y_{1}, \ldots, y_{n}\right) \rightarrow \bigwedge_{1 \leq i \leq n} \alpha^{\prime y_{i}}\left(\rho_{i}\right)\right)$;

$-\alpha^{\prime x}\left(\rho_{1} \odot \rho_{2}\right)=\alpha^{\prime x}\left(\rho_{1}\right) \odot \alpha^{\prime x}\left(\rho_{2}\right), \odot \in\{\vee, \wedge, \rightarrow\}$;

Lemma 1. For any $\Delta \in\left|\operatorname{Sign}^{\mathcal{H} \mathcal{I}}\right|, \rho \in \operatorname{Sen}^{\mathcal{F} \mathcal{O L}}(\Phi(\Sigma)), M^{\prime} \in \operatorname{Mod}^{\mathcal{F O} \mathcal{L}}\left(\Phi^{\prime}(\Delta)\right)$ and $s \in S$,

$$
M_{s}^{\prime} \models_{\Phi(\Sigma)} \rho \text { if and only if } M^{\prime s} \models_{\Phi^{\prime}(\Delta)+x} \alpha^{x}(\rho),
$$

where $M^{\prime s}$ denotes the expansion of $M^{\prime}$ to $\Phi^{\prime}(\Delta)+x$ defined by $x^{M^{\prime s}}=s$.

Theorem 3. For any $\Delta \in\left|\operatorname{Sign}^{\mathcal{H I}}\right|, \rho \in \operatorname{Sen}^{\mathcal{H I}}(\Delta) M^{\prime} \in \operatorname{Mod}^{\mathcal{F} \mathcal{O L}}\left(\Phi^{\prime}(\Delta)\right)$ and $s \in S$,

$$
\beta_{\Delta}^{\prime}\left(M^{\prime}\right)\left(\models_{\Delta}^{\mathcal{H} \mathcal{I}}\right)^{s} \rho \text { iff } M^{\prime s} \models_{\Phi^{\prime}(\Delta)+x}^{\mathcal{F} \mathcal{L}} \alpha_{\Delta}^{\prime x}(\rho),
$$

where $M^{\prime s}$ denotes the expansion of $M^{\prime}$ to $\Phi^{\prime}(\Delta)+x$ defined by $x^{M^{\prime s}}=s$.

Corollary 4 (Satisfaction condition for $\left.\left(\Phi^{\prime}, \alpha^{\prime}, \beta^{\prime}\right)\right) .\left(\Phi^{\prime}, \alpha^{\prime}, \beta^{\prime}\right)$ is comorphism $\mathcal{H} \mathcal{I} \rightarrow \mathcal{F} \mathcal{O L}$, i.e. for any $\Delta \in\left|\operatorname{Sign}^{\mathcal{H I}}\right|, \rho \in \operatorname{Sen}^{\mathcal{H I}}(\Delta)$ and $M^{\prime} \in$ $\operatorname{Mod}^{\mathcal{F O} \mathcal{L}}\left(\Phi^{\prime}(\Delta)\right)$,

$$
\beta_{\Delta}^{\prime}\left(M^{\prime}\right) \models_{\Delta}^{\mathcal{H} \mathcal{I}} \rho \text { if and only if } M^{\prime} \models_{\Phi^{\prime}(\Delta)}^{\mathcal{F} \mathcal{L}} \alpha_{\Delta}^{\prime}(\rho) .
$$

Example $5(\mathcal{H} \mathcal{E} \mathcal{Q} 2 \mathcal{F} \mathcal{O} \mathcal{L})$. A simple, but useful example of the construction proposed above arises by its application to the embedding of $\mathcal{E} \mathcal{Q} \mathcal{L}$ into $\mathcal{F O} \mathcal{L}$, entailing a comorphism $\mathcal{H} \mathcal{E} \mathcal{L} \rightarrow \mathcal{F O} \mathcal{L}$.

\section{Conclusions and further work}

The paper's contribution is twofold: first it defines a method to hybridize arbitrary institutions; then it is shown that a comorphism from an arbitrary institution to $\mathcal{F O} \mathcal{L}$ gives rise to another comorphism from its (quantifier-free) hybridization to $\mathcal{F O} \mathcal{L}$. 
Beyond their intrinsic theoretical interest, the application of these results seems promising. On the one hand, hybridization of logics is achieved, by this method, in a systematic way which applies to a broad class of logics. On the other, our second result contributes to add effective tool support to reasoning about hybrid specifications, by resorting to $\mathcal{F} \mathcal{O L}$-oriented verification tools.

This work also opens a number of interesting research directions. We discuss below the set of main topics in our agenda.

Remark 1. An aspect of our method, which increases the complexity of hybridizing arbitrary institutions, is the need for "desconstructing" the base institution. For instance, in order to hybridize $\mathcal{F O} \mathcal{L}$, we have to take in the role of a base institution its sub-institution of atomic formulas (without quantifiers and boolean connectives). The same happens in the hybridization of propositional logic (see Ex. 2). In order to overcome this situation, it is necessary to find a way to proscribe the overloading of connectives at the base and hybrid levels. The problem may be solved resorting to the (abstract) notion of boolean connective (cf. [14, Chap. 3]). For instance, suppose that the institution $\mathcal{I}$ has semantical negation, i.e., that for any $\rho \in \operatorname{Sen}^{\mathcal{I}}(\Sigma)$ there is a $\rho^{\prime} \in \operatorname{Sen}^{\mathcal{I}}(\Sigma)$ such that for any $\mathcal{M} \in\left|\operatorname{Mod}^{\mathcal{I}}(\Sigma)\right|, \mathcal{M} \models_{\Sigma}^{\mathcal{I}} \rho^{\prime}$ iff it is false that $\mathcal{M} \models_{\Sigma}^{\mathcal{I}} \rho$. Then, in order to avoid the connectives negation, we may replace, on the definition of the hybrid sentences, the negation introduction by

$$
\text { If } \rho \in \operatorname{Sen}^{\mathcal{H I}}(\Sigma, \operatorname{Nom}, \Lambda) \backslash \operatorname{Sen}^{\mathcal{I}}(\Sigma) \text {, then } \neg \rho \in \operatorname{Sen}^{\mathcal{H I}}(\Sigma, \operatorname{Nom}, \Lambda) \text {, }
$$

and similarly for the other boolean connectives. This seems to be enough to obtain the $\mathcal{H} \mathcal{F} \mathcal{O L}$ from $\mathcal{F O} \mathcal{L}$

Hybridization of modal logics is a more challenging question: how to introduction of nominals on institutions that already have Kripke semantics? For instance it is known that $\mathcal{C} \mathcal{T} \mathcal{L}$ defines an institution (cf. [9]) and that there are hybrid extensions of this logic currently being studied (cf. [25,20]). Actually, this sort of hybridization falls out of the scope of the the method discussed in this paper. Its application to $\mathcal{C} \mathcal{T} \mathcal{L}$ leads to a a kind of "graph of graphs", raising the question of how can such a double modalization be avoided. Apparently, there are tricky technical aspects to overcome. However, the hybridization of a (concrete) institution with Kripke semantics, i.e., the introduction of nominals and a satisfaction operator on a institution whose models are already of the form $\left(S,\left(M_{s}\right)_{s \in S}\right)$, seems to be an easy task. Hence, an answer for this problem resorts to the decomposition of the hybridization process into two steps: a modalization followed by a hybridization. The former, may be defined as in [16] just making a straightforward generalization to sets of modal symbols $\Lambda$. The latter is then applied to the resulting institution. to any institution with Kripke semantics.

Remark 2 (Calculus for hybrid institutions). Comparing the calculus of [7] for hybrid propositional logic with that of [4] for hybrid first-order logic, a common structure pops out: they "share" rules involving sentences with nominals and satisfaction operators (i.e., formulas with "hybrid nature") and have specific 
rules to reason about "atomic sentences" that come from the base institution. Hence, it makes sense to think on the development of a general proof calculus for hybrid institutions built on top of the calculus equipping the base institution in the style of $[3,11]$.

Remark 3 (Modal symbols quantification). Another interesting point to explore is the power of quantification over modal symbols, for instance by considering in the quantification space inclusions of the form $\Lambda \hookrightarrow \Lambda+\lambda$. Using this quantification it seems possible to express general properties about the state space of a model. For instance, we have that $\mathcal{P} \models^{s}(\forall \lambda) p \rightarrow[\lambda] p$ means that if $p$ holds in $s$ then it is invariant in all the model and $\mathcal{P} \models^{s}(\forall \lambda) p \rightarrow[\lambda] q$ to say that if $p$ holds on $s$ then $q$ holds in other state of the model.

Remark 4 (New case studies). There are many interesting hybrid institutions that may be obtained by application of the method proposed in this paper. Particularly interesting case studies are the derivation of both intuitionistic hybrid logic [10,6] and many-valued hybrid logic [19] from their respective bases.

Remark 5 (On encoding hybridizations to $\mathcal{F} \mathcal{O} \mathcal{L}$ ). An important property of logic encodings, which guarantees the sound and complete borrowing of formal reasoning from the target to the source of the encodings, is that they keep unchanged the consequence relation of the encoded logic (see Fact 2). In the case of the encoding $\mathcal{H} \mathcal{I} \rightarrow \mathcal{F O} \mathcal{L}$ defined as a comorphism in Section 4 this would have followed immediately if $\left(\Phi^{\prime}, \alpha^{\prime}, \beta^{\prime}\right): \mathcal{H} \mathcal{I} \rightarrow \mathcal{F O} \mathcal{L}$ were conservative which in turns should be a natural consequence of the conservativeness of $(\Phi, \alpha, \beta): \mathcal{I} \rightarrow \mathcal{F} \mathcal{O L}$. Unfortunately this latter step does not work in general, however this scheme may work if we extended our theory by considering also a 'rigid' part for the signatures and models as in [16].

Our current encoding of hybridizations to $\mathcal{F O} \mathcal{L}$ is limited in the applications by the fact that it applies only to encodings of the base institution that can be expressed as plain comorphisms to $\mathcal{F} \mathcal{O} \mathcal{L}$. This means that our current result may be in reality applied for hybridizations of various fragments of $\mathcal{F O} \mathcal{L}$ but none of the myriad of specification logics that are encoded into $\mathcal{F} \mathcal{O} \mathcal{L}$ by the so-called 'theoroidal comorphisms' [18]. We plan to extend our encoding result to this more general situation, thus widely enlarging the $\mathcal{F} \mathcal{O} \mathcal{L}$-oriented formal reasoning support for hybridized logics.

We also plan to extend or encoding result also to quantified hybridizations.

Remark 6 (Model theory for hybridized institutions). A deeper development of the model theory of a specification formalism always results into a better understanding of its specification power. Our general hybridization method opens the door for a general institution-independent approach to the model theory of hybrid(ized) logics by using techniques from [14]. We believe that the end result of such investigation would make yet another point in favour of the hybrid variants of modal logics, because we expect them to display better model theoretic properties than their non-hybrid variants. 
In particular we are thinking to extend the method of ultraproducts of [16] from modalized to hybridized institutions, to investigate a general method of diagrams and the existence of initial semantics for hybridized institutions. The latter has a special specification theoretic significance: it would give foundational support for classical algebraic specification style with hybrid(ized) logics. The method of diagrams, which is a very common model theoretic property of logics and a technique that pervades a lot of model theoretic results (see $[13,14]$ for its institution-independent expressions), unfortunately fails on modal logics. However because of the special "hybrid features" we expect it to hold in some form in hybrid(ized) logics.

\section{References}

1. C. Areces, P. Blackburn, and S. R. Delany. Bringing them all together. Journal of Logic and Computation, 11:657-669, 2001.

2. P. Blackburn. Representation, reasoning, and relational structures: a hybrid logic manifesto. Logic Journal of IGPL, 8:2000, 2000.

3. T. Borzyszkowski. Logical systems for structured specifications. Theoretical Computer Science, 286(2):197-245, 2002.

4. T. Braüner. Natural deduction for first-order hybrid logic. Journal of Logic, Language and Information, 14:173, 2005.

5. T. Braüner. Hybrid Logic and its Proof-Theory, volume 37 of Applied Logic Series. Springer, 2011.

6. T. Braüner and V. de Paiva. Intuitionistic hybrid logic. J. Applied Logic, 4(3):231$255,2006$.

7. T. Braüner. Natural deduction for first-order hybrid logic. Journal of Logic, Language and Information, 14:173, 2005.

8. R. Burstall and J. Goguen. The semantics of Clear, a specification language. In D. Bjorner, editor, 1979 Copenhagen Winter School on Abstract Software Specification, volume 86 of Lecture Notes in Computer Science, pages 292-332. Springer, 1980.

9. M. V. Cengarle. The temporal logic institution. Technical report, Universitat Munchen, Insitut fur informatik, 1998.

10. R. Chadha, D. Macedonio, and V. Sassone. A hybrid intuitionistic logic: Semantics and decidability. Journal of Logic and Computation, 16:2006, 2005.

11. M. Codescu and D. Găină. Birkhoff completeness in institutions. Logica Universalis, 2(2):277-309, 2008.

12. S. Demri, R. Lazic, and D. Nowak. On the freeze quantifier in constraint ltl: decidability and complexity. Technical Report LSV-05-03, Laboratoire Specification et Verification, 2005.

13. R. Diaconescu. Elementary diagrams in institutions. Journal of Logic and Computation, 14(5):651-674, 2004.

14. R. Diaconescu. Institution-independent Model Theory. Birkhäuser Basel, 2008.

15. R. Diaconescu. Quasi-boolean encodings and conditionals in algebraic specification. Journal of Logic and Algebraic Programming, 79(2):174-188, 2010.

16. R. Diaconescu and P. S. Stefaneas. Ultraproducts and possible worlds semantics in institutions. Theor. Comput. Sci., 379(1-2):210-230, 2007. 
17. J. Goguen and R. Burstall. Institutions: Abstract model theory for specification and programming. Journal of the Association for Computing Machinery, 39(1):95$146,1992$.

18. J. Goguen and G. Roşu. Institution morphisms. Formal Aspects of Computing, 13:274-307, 2002.

19. J. Hansen, T. Bolander, and T. Braüner. Many-valued hybrid logic. In C. Areces and R. Goldblatt, editors, Advances in Modal Logic, pages 111-132. College Publications, 2008

20. A. Kara, M. Lange, T. Schwentick, and V. Weber. On the hybrid extension of CTL and CTL+. CoRR, abs/0906.2541, 2009.

21. M. Martins, A. Madeira, and L. Barbosa. Reasoning about complex requirements in a uniform setting. Technical report, DI-CCTC-2-1-2011, 2011.

22. S. Passy and T. Tinchev. An essay in combinatory dynamic logic. Inf. Comput., 93:263-332, August 1991.

23. A. N. Prior. Past, Present and Future. Oxford University Press, 1967.

24. U. Sattler and M. Y. Vardi. The hybrid $\mu$-calculus. In R. Goré, A. Leitsch, and T. Nipkow, editors, IJCAR, volume 2083 of Lecture Notes in Computer Science, pages 76-91. Springer, 2001.

25. V. Weber. On the complexity of branching-time logics. In E. Grädel and R. Kahle, editors, CSL, volume 5771 of Lecture Notes in Computer Science, pages 530-545. Springer, 2009. 


\section{Appendix: Proofs}

Corollary 1. $\operatorname{Sign}^{\mathcal{H I}}$ has all small co-limits.

Proof. As $\operatorname{Sign}^{\mathcal{R E L}}$ is isomorphic to the power category $\operatorname{Set}^{\omega}$ (with $\omega$ being the set of the natural numbers) and Set has small co-limits it follows that $\operatorname{Sign}^{\mathcal{R E} \mathcal{L}}$ has small co-limits. From this and from Prop. 1 follows the conclusion of the corollary.

Proposition 2. $\operatorname{Sen}^{\mathcal{H} \mathcal{I}}$ is a functor $\operatorname{Sign}^{\mathcal{H I}} \rightarrow$ Set.

Proof. The functoriality of $\operatorname{Sen}^{\mathcal{H I}}$ follows rather straightforwardly by recursion on the structure of the sentences. Note that the recursion step corresponding to quantifiers relies crucially upon the properties of $\mathcal{D}^{\mathcal{H I}}$ being a quantification space.

Theorem 1. A pushout square of $\mathcal{H} \mathcal{I}$-signature morphisms is a (weak) amalgamation square (for $\operatorname{Mod}^{\mathcal{H} \mathcal{I}}$ ) if the underlying square of signature morphisms in $\mathcal{I}$ is a (weak) amalgamation square.

Proof. The amalgamation of $\left(M_{1}, R_{1}\right)$ and $\left(M_{2}, R_{2}\right)$ is $\left(M^{\prime}, R^{\prime}\right)$ defined as follows:

- $R^{\prime}$ is an amalgamation of $R_{1}$ and $R_{2}$ is $\mathcal{R E \mathcal { L }}$; this is because the projection functor $\operatorname{Sign}^{\mathcal{H} \mathcal{I}}=\operatorname{Sign}^{\mathcal{I}} \times \operatorname{Sign}^{\mathcal{R E L}} \rightarrow \operatorname{Sign}^{\mathcal{R} \mathcal{L} \mathcal{L}}$ preserves pushouts, hence the underlying square of signature morphisms in $\mathcal{R E} \mathcal{L}$ is pushout.

- Let $|R|$ be the carrier set of $R_{1}, R_{2}$ and $R$ (by definition of reduct note all of them share the same carrier set). Then $M^{\prime}:|R| \rightarrow\left|\operatorname{Mod}^{\mathcal{I}}\left(\Sigma^{\prime}\right)\right|$ is the function corresponding to the (weak) pullback property of functor $\left|\operatorname{Mod}^{\mathcal{I}}(-)\right|$ image of the underlying square of signature morphisms in $\mathcal{I}$. This, by Prop. 1 , is a pushout square, as well. The (weak) model amalgamation assumption for $\mathcal{I}$ means that $\left|\operatorname{Mod}^{\mathcal{I}}(-)\right|$ maps this to a (weak) pullback.

Theorem 2. Let $\Delta=(\Sigma$, Nom, $\Lambda)$ and $\Delta^{\prime}=\left(\Sigma^{\prime}, \mathrm{Nom}^{\prime}, \Lambda^{\prime}\right)$ be two $\mathcal{H}$ signatures and $\varphi: \Delta \rightarrow \Delta^{\prime}$ a morphism of signatures. For any $\rho \in \operatorname{Sen}^{\mathcal{H I}}(\Delta)$, $\left(M^{\prime}, R^{\prime}\right) \in\left|\operatorname{Mod}^{\mathcal{H I}}\left(\Delta^{\prime}\right)\right|$, and $s \in|R|$

$$
\operatorname{Mod}^{\mathcal{H I}}(\varphi)\left(M^{\prime}, R^{\prime}\right) \models{ }^{s} \rho \text { iff }\left(M^{\prime}, R^{\prime}\right) \models^{s} \operatorname{Sen}^{\mathcal{H I}}(\varphi)(\rho) .
$$

Proof. Let us denote $\operatorname{Mod}^{\mathcal{H I}}\left(M^{\prime}, R^{\prime}\right)=\langle M, R\rangle$. The proof is by recursion on the structure of sentences:

\section{Atomic formulas:}


If $\rho=i$ for some $i \in$ Nom:

$$
\begin{aligned}
& \operatorname{Mod}^{\mathcal{H I}}(\varphi)\left(M^{\prime}, R^{\prime}\right) \models^{s} i \\
\Leftrightarrow & \quad\left\{\text { definition of } \models^{\mathcal{H}}\right\} \\
& i^{R}=s \\
\Leftrightarrow & \quad\left\{\text { by definition of reduct } \varphi_{\text {Nom }}(i)^{R^{\prime}}=i^{R}\right\} \\
& \left(M^{\prime}, R^{\prime}\right) \models^{s} \varphi_{\text {Nom }}(i) \\
\Leftrightarrow & \quad\left\{\text { by definition of } \operatorname{Sen}^{\mathcal{H I}}(\varphi)\right\} \\
& \left(M^{\prime}, R^{\prime}\right) \models^{s} \operatorname{Sen}^{\mathcal{H I}}(\varphi)(i)
\end{aligned}
$$

If $\rho \in \operatorname{Sen}^{\mathcal{I}}(\Sigma)$ :

$$
\begin{aligned}
& \operatorname{Mod}^{\mathcal{H I}}(\varphi)\left(M^{\prime}, R^{\prime}\right) \models^{s} \rho \\
\Leftrightarrow & \quad\left\{\text { definition of } \models^{\mathcal{H I}}\right\} \\
& \operatorname{Mod}^{\mathcal{I}}\left(\varphi_{\text {Sig }}\right)\left(M_{s}^{\prime}\right)=M_{s} \models^{\mathcal{I}} \rho \\
\Leftrightarrow & \quad\{\text { since } \mathcal{I} \text { is an institution }\} \\
& M_{s}^{\prime} \models \operatorname{Sen}{ }^{\mathcal{I}}\left(\varphi_{\text {Sig }}\right)(\rho) \\
\Leftrightarrow & \quad\left\{\text { by definition of } \models^{\mathcal{H I}} \text { and of } \operatorname{Sen}^{\mathcal{H I}}(\varphi)\right\} \\
& \left(M^{\prime}, R^{\prime}\right) \models^{s} \operatorname{Sen}^{\mathcal{H I}}(\varphi)(\rho)
\end{aligned}
$$

\section{Composed formulas:}

If $\rho$ is of form $\xi \vee \xi^{\prime}$ for some $\xi, \xi^{\prime} \in \operatorname{Sen}^{\mathcal{H I}}(\Delta)$ :

$$
\begin{aligned}
& \operatorname{Mod}^{\mathcal{H I}}(\varphi)\left(M^{\prime}, R^{\prime}\right) \models^{s} \xi \vee \xi^{\prime} \\
\Leftrightarrow & \quad\left\{\text { definition of } \models^{\mathcal{H} \mathcal{I}}\right\} \\
& \operatorname{Mod}^{\mathcal{H I}}(\varphi)\left(M^{\prime}, R^{\prime}\right) \models^{s} \xi \operatorname{or}_{\operatorname{Mod}^{\mathcal{H I}}(\varphi)\left(M^{\prime}, R^{\prime}\right) \models^{s} \xi^{\prime}} \quad\{\quad\{\text { I.H }\} \\
& \left(M^{\prime}, R^{\prime}\right) \models^{s} \operatorname{Sen}^{\mathcal{H I}}(\varphi)(\xi) \text { or }\left(M^{\prime}, R^{\prime}\right) \models^{s} \operatorname{Sen}^{\mathcal{H I}}(\varphi)\left(\xi^{\prime}\right) \\
\Leftrightarrow & \quad\left\{\text { by definition of } \models^{\mathcal{H I}}\right\} \\
& \left(M^{\prime}, R^{\prime}\right) \models^{s} \operatorname{Sen}^{\mathcal{H I}}(\varphi)\left(\xi \vee \xi^{\prime}\right)
\end{aligned}
$$

The proofs for connectives $\{\wedge, \rightarrow, \neg\}$ are analogous. 
If $\rho$ is of form $[\lambda]\left(\xi_{1}, \ldots, \xi_{n}\right)$, for some $\xi_{1}, \ldots, \xi_{n} \in \operatorname{Sen}^{\mathcal{H I}}(\Delta), \lambda \in \Lambda_{n+1}$ :

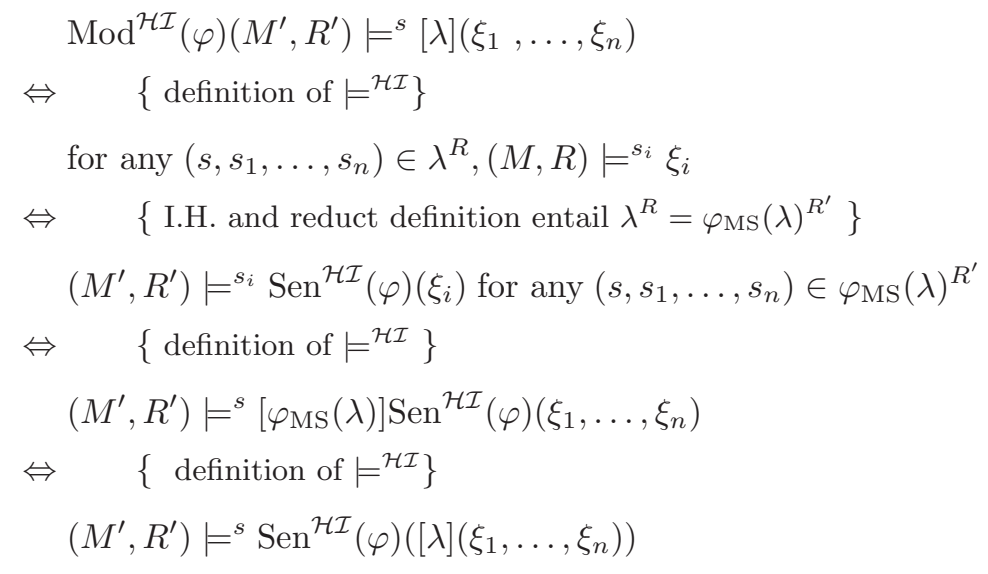

If $\rho$ is of form $@_{i} \xi$ for some $\xi \in \operatorname{Sen}^{\mathcal{H} \mathcal{I}}(\Delta), i \in$ Nom:

$$
\begin{aligned}
& \operatorname{Mod}^{\mathcal{H I}}(\varphi)\left(M^{\prime}, R^{\prime}\right) \models^{s} @_{i} \xi \\
& \Leftrightarrow \quad\left\{\text { definition of } \models^{\mathcal{H} I}\right. \text { \} } \\
& \operatorname{Mod}^{\mathcal{H I}}(\varphi)\left(M^{\prime}, R^{\prime}\right) \models^{i^{R}} \xi \\
& \Leftrightarrow \quad\{\text { I.H. }\} \\
& \left(M^{\prime}, R^{\prime}\right) \models^{i^{R}} \operatorname{Sen}^{\mathcal{H I}}(\varphi)(\xi) \\
& \Leftrightarrow \quad\left\{\text { since by reduct definition, } i^{R}=\varphi_{\mathrm{Nom}}(i)^{R^{\prime}}\right\} \\
& \left(M^{\prime}, R^{\prime}\right) \models^{\varphi_{\text {Nom }}(i)^{R^{\prime}}} \operatorname{Sen}^{\mathcal{H} \mathcal{I}}(\varphi)(\xi) \\
& \Leftrightarrow \quad\{\text { definition of satisfaction for @ }\} \\
& \left(M^{\prime}, R^{\prime}\right) \models^{s} @_{\varphi_{\mathrm{Nom}}(i)} \operatorname{Sen}^{\mathcal{H I}}(\varphi)(\xi) \\
& \Leftrightarrow \quad\left\{\operatorname{Sen}^{\mathcal{H I}}(\varphi) \text { definition }\right\} \\
& \left(M^{\prime}, R^{\prime}\right) \models^{s} \operatorname{Sen}^{\mathcal{H I}}(\varphi)\left(@_{i} \xi\right)
\end{aligned}
$$


If $\rho$ is of form $\left(\forall \chi: \Delta \rightarrow \Delta_{1}\right) \rho$ :

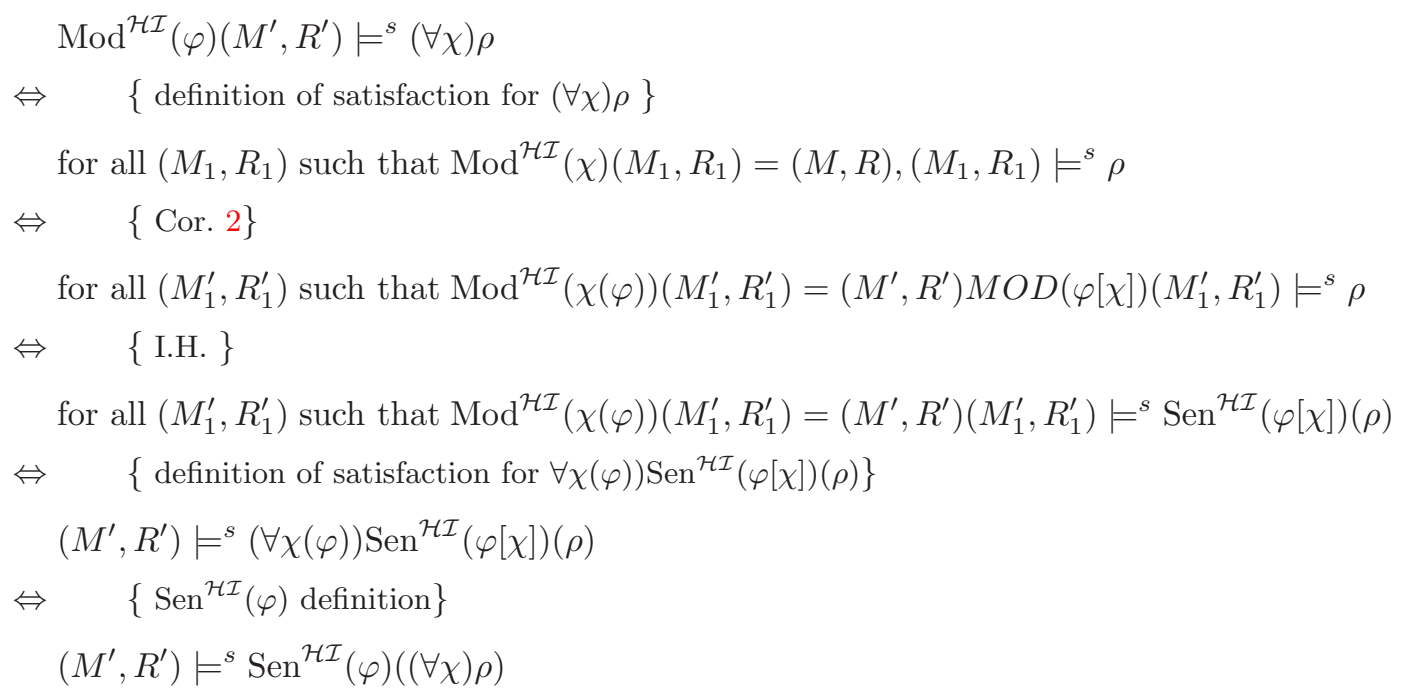

Lemma 1. For any $\Delta \in\left|\operatorname{Sign}^{\mathcal{H} \mathcal{I}}\right|, \rho \in \operatorname{Sen}^{\mathcal{F O} \mathcal{L}}(\Phi(\Sigma)), M^{\prime} \in \operatorname{Mod}^{\mathcal{F O} \mathcal{L}}\left(\Phi^{\prime}(\Delta)\right)$ and $s \in S$,

$$
M_{s}^{\prime} \models_{\Phi(\Sigma)} \rho \text { if and only if } M^{\prime s} \models_{\Phi^{\prime}(\Delta)+x} \alpha^{x}(\rho),
$$

where $M^{\prime s}$ denotes the expansion of $M^{\prime}$ to $\Phi^{\prime}(\Delta)+x$ defined by $x^{M^{\prime s}}=s$.

Proof. The proof is by recursion on the structure of $\rho$.

If $\rho$ is $t \approx t^{\prime}$ : the proof for this case is an immediate consequence of the following relation

$$
t^{M_{s}^{\prime}}=\alpha^{x}(t)^{M^{\prime s}} \quad(\text { for } t \text { any term })
$$


which is proved by recursion on $t$ as follows:

$$
\begin{aligned}
& f\left(t_{1}, \ldots, t_{n}\right)^{M_{s}^{\prime}} \\
= & \quad\{\text { definition of evaluation of terms }\} \\
& f^{M_{s}^{\prime}}\left(t_{1}^{M_{s}^{\prime}}, \ldots, t_{n}^{M_{s}^{\prime}}\right) \\
= & \quad\left\{\text { definition of } f^{M_{s}^{\prime}}\right\} \\
& f^{M^{\prime}}\left(s, t_{1}^{M_{s}^{\prime}}, \ldots, t_{n}^{M_{s}^{\prime}}\right) \\
= & \quad\{\text { I.H. }\} \\
& f^{M^{\prime}}\left(s, \alpha^{x}\left(t_{1}\right)^{M^{\prime s}}, \ldots, \alpha^{x}\left(t_{n}\right)^{M^{\prime s}}\right) \\
= & \quad\left\{\text { because } x^{M^{\prime s}}=s \text { and definition of term evaluation }\right\} \\
& f\left(x, \alpha^{x}\left(t_{1}\right), \ldots, \alpha^{x}\left(t_{n}\right)\right)^{M^{\prime s}} \\
= & \quad\left\{\text { definition of } \alpha^{x}\right\} \\
& \alpha^{x}\left(f\left(t_{1}, \ldots, t_{n}\right)\right)^{M^{\prime s}}
\end{aligned}
$$

If $\rho$ is $\pi\left(t_{1}, \ldots, t_{n}\right)$ :

$$
\begin{aligned}
& M_{s}^{\prime} \models \pi\left(t_{1}, \ldots, t_{n}\right) \\
\Leftrightarrow & \quad\{\text { definition } \mathcal{F} \mathcal{O L} \text { satisfaction }\} \\
& \left(t_{1}^{M_{s}^{\prime}}, \ldots, t_{n}^{M_{s}^{\prime}}\right) \in \pi^{M_{s}^{\prime}} \\
\Leftrightarrow & \quad\left\{\text { definition of } \pi^{M_{s}^{\prime}} \text { and by }(8)\right\} \\
& \left(s, \alpha^{x}\left(t_{1}\right)^{M^{\prime s}}, \ldots, \alpha^{x}\left(t_{n}\right)^{M^{\prime s}}\right) \in \pi^{M^{\prime}} \\
\Leftrightarrow & \quad\left\{\text { because } x^{M^{\prime s}}=s \text { and definition of } \alpha^{x}\left(\pi\left(t_{1}, \ldots, t_{n}\right)\right)\right\} \\
& M^{\prime s} \models \pi\left(x, \alpha^{x}\left(t_{1}\right), \ldots, \alpha^{x}\left(t_{n}\right)\right)=\alpha^{x}\left(\pi\left(t_{1}, \ldots, t_{n}\right)\right)
\end{aligned}
$$

If $\rho$ is a composed sentence: the proof reduces to a direct application of the induction hypothesis.

Theorem 3. For any $\Delta \in\left|\operatorname{Sign}^{\mathcal{H} \mathcal{I}}\right|, \rho \in \operatorname{Sen}^{\mathcal{H I}}(\Delta) M^{\prime} \in \operatorname{Mod}^{\mathcal{F} \mathcal{O L}}\left(\Phi^{\prime}(\Delta)\right)$ and $s \in S$,

$$
\beta_{\Delta}^{\prime}\left(M^{\prime}\right)\left(\models_{\Delta}^{\mathcal{H} \mathcal{I}}\right)^{s} \rho \text { iff } M^{\prime s} \models_{\Phi^{\prime}(\Delta)+x}^{\mathcal{F} \mathcal{O}} \alpha_{\Delta}^{\prime x}(\rho),
$$

where $M^{\prime s}$ denotes the expansion of $M^{\prime}$ to $\Phi^{\prime}(\Delta)+x$ defined by $x^{M^{\prime s}}=s$.

Proof. The proof is by recursion on the structure of $\rho$. Let us denote $\beta_{\Delta}\left(M^{\prime}\right)$ by $\left(M^{\prime \prime}, R^{\prime}\right)$. 
If $\rho=i$ for some $i \in$ Nom:

$$
\begin{aligned}
& \beta_{\Delta}^{\prime}\left(M^{\prime}\right) \models_{{ }_{\Delta}}^{s} i \\
\Leftrightarrow & \quad\left\{\text { definition of } \models^{\mathcal{H} \mathcal{I}}\right\} \\
& i^{R^{\prime}}=s \\
\Leftrightarrow & \quad\left\{\text { by definition of } \beta^{\prime} \text { and of } M_{s}^{\prime}\right\} \\
& i^{M^{\prime}}\left(=i^{M^{\prime s}}\right)=x^{M^{\prime s}} \\
\Leftrightarrow \quad & \{\} \\
& M^{\prime s} \models_{\Phi^{\prime}(\mathcal{L})+x}^{\mathcal{F} \mathcal{L}} i \approx x=\alpha^{\prime x}(i)
\end{aligned}
$$

If $\rho \in \operatorname{Sen}^{\mathcal{I}}(\Sigma)$ :

$$
\begin{aligned}
& \beta_{\Delta}^{\prime}\left(M^{\prime}\right) \models_{\Delta}^{s} \rho \\
\Leftrightarrow & \quad\left\{\text { definition of } \models^{\mathcal{H} \mathcal{I}} \text { and of } \beta^{\prime}\right\} \\
& \beta_{\Sigma}\left(M_{s}^{\prime}\right) \models_{\Sigma}^{\mathcal{I}} \rho \\
\Leftrightarrow & \quad\{\text { by the satisfaction condition of the comorphism }(\Phi, \alpha, \beta)\} \\
& M_{s}^{\prime} \models^{\mathcal{F} \mathcal{F} \mathcal{L}(\Sigma)} \alpha_{\Sigma}(\rho) \\
\Leftrightarrow & \quad\{\text { Lemma } 1\} \\
& M^{\prime s} \models_{\Phi^{\prime}(\mathcal{I})+x}^{\mathcal{F} \mathcal{L}} \alpha^{x}\left(\alpha_{\Sigma}(\rho)\right)=\alpha^{\prime x}(\rho)
\end{aligned}
$$

If $\rho=@_{i} \varphi$ :

$$
\begin{aligned}
& \beta_{\Delta}^{\prime}\left(M^{\prime}\right)\left(\models^{\mathcal{H I}}\right)^{s} @_{i} \rho \\
& \Leftrightarrow \quad\left\{\text { definition of } \models^{\mathcal{H I}}\right. \text { \} } \\
& \beta_{\Delta}^{\prime}\left(M^{\prime}\right)\left(\models \models^{\mathcal{H I}}\right)^{i^{R^{\prime}}} \rho \\
& \Leftrightarrow \quad\left\{\text { since } R^{\prime}=M^{\prime} \Gamma_{\langle\{\mathrm{ST}\}, \overline{\mathrm{Nom}}, \bar{\Lambda}\rangle}\right\} \\
& \beta_{\Delta}^{\prime}\left(M^{\prime}\right)(\models \mathcal{H I})^{i^{M^{\prime}}} \rho \\
& \Leftrightarrow \quad\{\text { I.H. }\} \\
& \left(M^{\prime}\right)^{i^{M^{\prime}}} \models_{\Phi^{\prime}(\Delta)+x}^{\mathcal{F} \mathcal{L}} \alpha^{\prime x}(\rho) \\
& \Leftrightarrow \quad\{\} \\
& M^{\prime} \models_{\Phi^{\prime}(\Delta)}^{\mathcal{F} \mathcal{L}} \alpha^{\prime i}(\rho) \\
& \Leftrightarrow \quad\left\{\alpha^{\prime} \text { definition }\right\} \\
& M^{\prime s} \models_{\Phi^{\prime}(\Delta)+x}^{\mathcal{F} \mathcal{L}} \alpha^{\prime x}\left(@_{i} \rho\right)
\end{aligned}
$$



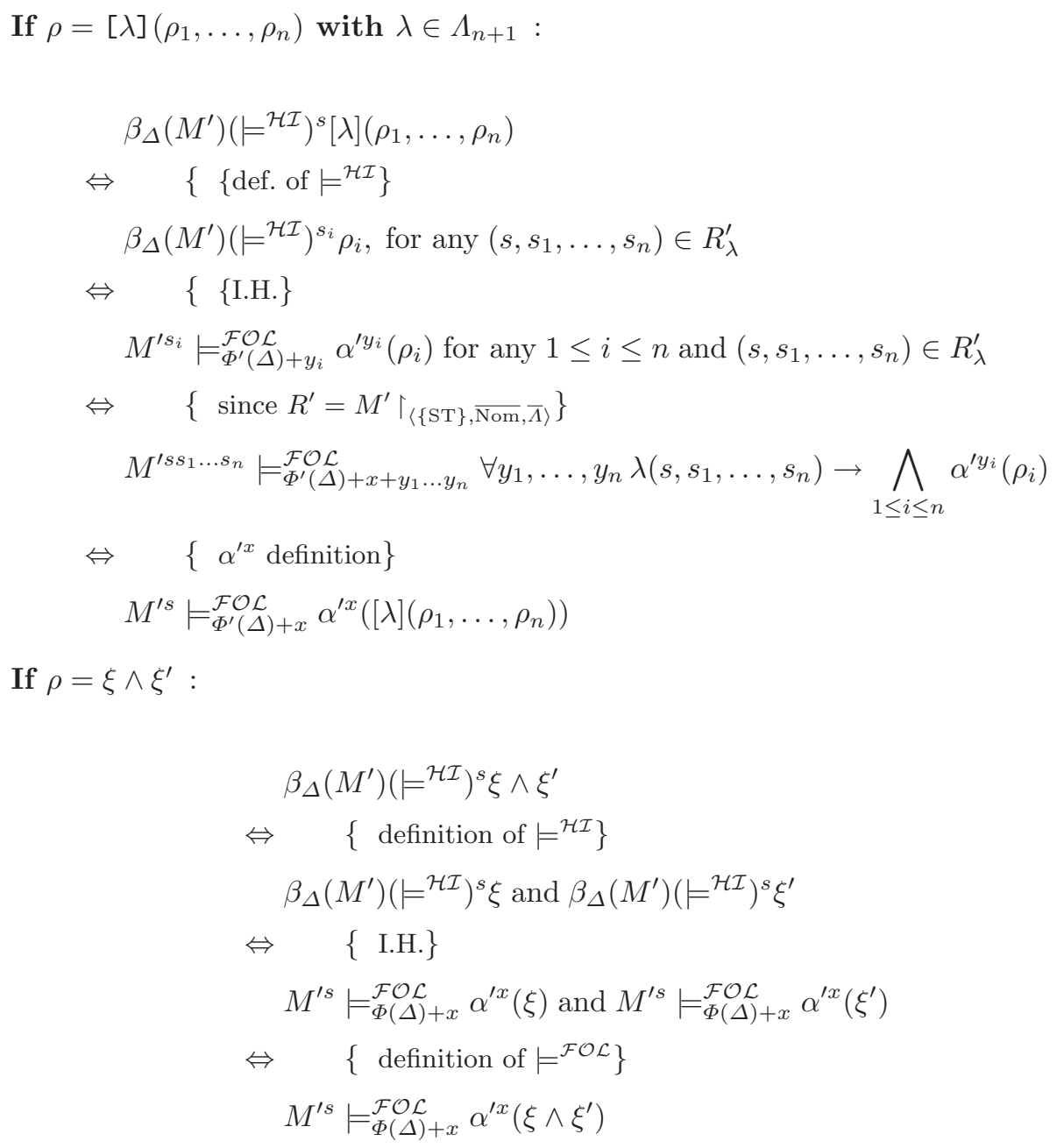

The proofs for connectives $\{\vee, \neg \rightarrow\}$ are analogous. 